\title{
Harrison-Zel'dovich primordial spectrum is consistent with observations
}

\author{
Stefania Pandolfi, ${ }^{1}$ Asantha Cooray, ${ }^{2}$ Elena Giusarma, ${ }^{3}$ \\ Edward W. Kolb, ${ }^{4}$ Alessandro Melchiorri, ${ }^{5}$ Olga Mena ${ }^{3}$, and Paolo Serra ${ }^{2}$ \\ ${ }^{1}$ ICRA and INFN, Universita' di Roma "La Sapienza", Ple. Aldo Moro 2, 00185, Rome, Italy \\ ${ }^{2}$ Center for Cosmology, Department of Physics $\& 3$ Astronomy, \\ University of California, Irvine, California 92697, USA \\ ${ }^{3}$ IFIC, Universidad de Valencia-CSIC, 46071, Valencia, Spain \\ ${ }^{4}$ Department of Astronomy $\&$ Astrophysics, Enrico Fermi Institute, \\ and Kavli Institute for Cosmological Physics, University of Chicago, Chicago, Illinois 60637, USA \\ ${ }^{5}$ Physics Department and INFN, Universita' di Roma "La Sapienza", Ple. Aldo Moro 2, 00185, Rome, Italy

\begin{abstract}
Inflation predicts primordial scalar perturbations with a nearly scale-invariant spectrum and a spectral index approximately unity (the Harrison-Zel'dovich (HZ) spectrum). The first important step for inflationary cosmology is to check the consistency of the HZ primordial spectrum with current observations. Recent analyses have claimed that a HZ primordial spectrum is excluded at more than $99 \%$ c.l. Here we show that the HZ spectrum is only marginally disfavored if one considers
\end{abstract} \\ a more general reionization scenario. Data from the Planck mission will settle the issue. \\ PACS numbers: 98.80.-k 95.85.Sz, 98.70.Vc, 98.80.Cq
}

One usually models the dynamics of primordial inflation by the dynamics of a scalar field, know as the inflaton, evolving under the influence of a scalar potential. The scalar and tensor perturbation spectra produced by inflation depend upon the value of the inflaton potential during inflation and how rapidly the scalar field evolves during inflation. Observational information of the spectra can be translated into information about the inflaton potential, thus about physics far beyond the scales of the standard model of particle physics [1].

Cosmic microwave background (CMB) observables that map onto the the potential and its slope are 1) $n$, the spectral index of comoving scalar perturbations $P_{\mathcal{R}}$ : $n-1=d \ln P_{\mathcal{R}} / d \ln k$, and 2) $r$, the ratio of tensor (gravitational wave) perturbations to $P_{\mathcal{R}}$ [2].

$\mathrm{CMB}$ temperature and polarization anisotropies are the most promising route to explore the physics behind inflation. Recent measurements by the Wilkinson Microwave Anisotropy Probe (WMAP) satellite seven-year mission [3], combined with the ground-based and balloonborne experiments such as BOOMERanG [4], QUAD [5], ACBAR [6], and BICEP [7] have sharpened our knowledge of some key inflationary parameters.

With regard to the dynamics of inflation, a hotly debated question is whether the case of $n=1$ is significantly excluded by current observations (see e.g., Refs. [3]-[13]).

The scalar spectral index, $n$, has been recently constrained to the value $n=0.963 \pm 0.014$ at $68 \%$ c.l. by the WMAP seven-year dataset (WMAP7) [3] , disfavoring the value of $n=1$ at about two standard deviations. A combined analysis with galaxy clustering data gives $n=0.963 \pm 0.012$ at $68 \%$ c.l., ruling out $n=1$ at more than three standard deviations ([3], [13] ).

Compelling evidence that $n \neq 1$ would be quite revealing for two reasons. First, it would provide an indication for the dynamical evolution of the expansion rate of the universe as perturbations are being produced. Secondly, in many models of slow-roll inflation the amplitude of the tensor perturbations are proportional to $|n-1|$. Thus, the larger the departure of $n$ from unity, the more likely tensor perturbations would be within observational reach.

As it is well known, a scalar spectral index with an exact value of $n=1$ corresponds to the phenomenological model proposed by Harrison, Zel'dovich, and Peebles 14]. ${ }^{1}$ While one can construct inflationary models that give $n=1$ either approximately [15] or exactly [16], they are less than compelling. In fact, observations pointing to $n$ exactly unity may indicate that the origin of cosmic perturbations lies in some unknown fundamental process and may not arise from inflation.

In this paper we show that the value of $n$, and its uncertainty, derived from CMB datasets are sensitive to how one models the reionization of the universe. In particular, the statement that $n=1$ is observationally excluded is very much weakened if one treats reionization in a general manner.

It is well known from a large set of astrophysical observables that after primordial recombination (which occurred at a redshift of $z \sim 1100$ ) the universe "reionized" at a redshift $z>6$. Current constraints from CMB data assume a sudden and complete reionization at a redshift $z_{r}$. This parameter is usually assumed to be in the range $4<z_{r}<32$, and the constraints on $n$ are obtained after marginalization over $z_{r}$. The electron ionization fraction $x_{e}(z)$ is parametrized by $z_{r}$ such that for $z \ll z_{r}$ $x_{e}(z)=1\left(x_{e}(z)=1.08\right.$ for $z<3$ in order to take into account Helium recombination) and $x_{e}(z)=2 \times 10^{-4}$ for

\footnotetext{
${ }^{1}$ For the purpose of our paper, we will assume that the tensor modes are small enough as to be irrelevant.
} 
$z>z_{r}$, i.e., joining the value after primordial recombination with a smooth interpolation. We refer to this common procedure in what follows as "sudden" reionization.

However, the precise details of the reionization process are not very well known, and therefore the reionization history of the universe at those redshifts could have easily been very different.

As already remarked in Refs. [17] and [18], the assumption of a more general reionization scheme could affect the cosmological constraints on $n$. It is therefore timely, especially in view of the recent CMB and galaxy clustering data that have improved the bound on $n$, to investigate the impact of the reionization history on the current constraints on $n$ in a more general reionization scenario.

We adopt two methods for parametrizing the reionization history. The first method, developed in Ref. [17], is based on principal components that provide a complete basis for describing the effects of reionization on largescale $E$-mode polarization. Following Ref. [17], one can parametrize the reionization history as a free function of redshift by decomposing $x_{e}(z)$ into its principal components:

$$
x_{e}(z)=x_{e}^{f}(z)+\sum_{\mu} m_{\mu} S_{\mu}(z)
$$

where the principal components, $S_{\mu}(z)$, are the eigenfunctions of the Fisher matrix that describes the dependence of the polarization spectra on $x_{e}(z)$ (again, see Ref. [17]), $m_{\mu}$ are the amplitudes of the principal components for a particular reionization history, and $x_{e}^{f}(z)$ is the WMAP fiducial model at which the Fisher matrix is computed and from which the principal components are obtained. In what follows we use the publicly available $S_{\mu}(z)$ functions and varied the amplitudes $m_{\mu}$ for $\mu=1, \ldots, 5$ for the first five eigenfunctions. Hereafter we refer to this method as the $\mathrm{MH}$ (Mortonson- $\mathrm{Hu}$ ) case.

In the second approach, we use a different parametrization, sampling the evolution of the ionization fraction $x_{e}$ as a function of the redshift $z$ at 7 points $(z=$ $9,12,15,18,21,24,27)$, and interpolating the value of $x_{e}(z)$ between them with a cubic spline. For $30<z$ we fix $x_{e}=2 \times 10^{-4}$ as the value of $x_{e}$ expected before reionization (and after primordial recombination), while $x_{e}=1$ for $3<z<6$ and $x_{e}=1.08$ for $z<3$ in order to be in agreement with both Helium ionization and GunnPeterson test observations. This approach is very similar to the one used in Ref. [18], and we will refer to it as the LWB (Lewis-Weller-Battye) case.

Exploiting two different and more general reionization schemes will provide us with not only a consistency check of the results, but also a broader set of reionization histories. Finally, we also consider here the standard reionization model, i.e., sudden reionization.

We have then modified the Boltzmann CAMB code [19] incorporating the two generalized reionization sce- narios and extracted cosmological parameters from current data using a Monte Carlo Markov Chain (MCMC) analysis based on the publicly available MCMC package cosmomc 20].

We consider here a flat $\Lambda$ CDM universe described by a set of cosmological parameters

$$
\left\{\omega_{b}, \omega_{c}, \Theta_{s}, n, \log \left[10^{10} A_{s}\right]\right\},
$$

where $\omega_{b} \equiv \Omega_{b} h^{2}$ and $\omega_{c} \equiv \Omega_{c} h^{2}$ are the physical baryon and cold dark matter densities relative to the critical density, $\Theta_{s}$ is the ratio of the sound horizon to the angular diameter distance at decoupling, $A_{s}$ is the amplitude of the primordial spectrum, and $n$ is the scalar spectral index. In one case we will also consider the possibility of a redshift-dependent dark energy component evolving with equation of state $w(z)=w_{0}+w_{z} z /(1+z)$.

The extra parameters needed to describe the reionization are the five amplitudes of the eigenfunctions for the $\mathrm{MH}$ case, or the five amplitudes in the five bins for the LWB case, and one single common parameter, the optical depth, $\tau$, for the sudden reionization case.

Our basic data set is the seven-year WMAP data [3] (temperature and polarization) with the routine for computing the likelihood supplied by the WMAP team. Together with the WMAP data, we also augment the WMAP7 data with the CMB datasets from BOOMERanG [4], QUAD [5], ACBAR [6], and BICEP 7]. For all these experiments we marginalize over a possible contamination from the Sunyaev-Zeldovich component, rescaling the WMAP template at the corresponding experimental frequencies. We therefore consider two cases: we first analyze the WMAP data alone, referring to it as to the "WMAP7" case, and we then include the remaining CMB experiments ("CMB All"). Finally we also consider, separately, the galaxy clustering results from LRG-7 of Ref. 21] and the baryonic acoustic oscillation (BAO) data from the same dataset, see Ref. 22].

Our main results are reported in Table I, where we list the constraints on $n$ for the different reionization scenarios using different datasets. Notice that using the $\mathrm{MH}$ parametrization drastically alters the constraints on $n$. For the WMAP7 case alone, the HZ spectrum is not only in agreement with the data, but is also close to the best-fit value. When the remaining CMB experiments are included, the best-fit value of $n$ shifts to lower values, but it is still consistent at better than $95 \%$ c.l. with $n=1$. When information from galaxy clustering is included, a value of $n=1$ is excluded at about the $95 \%$ c.l., i.e., in a much less conclusive way than in the sudden case where $n=1$ is excluded at more than $99.7 \%$ c.l. (see e.g., Ref. [13]).

Adopting the LWB parameterization of reionization has very similar effects. The HZ spectrum is only marginally excluded at a $95 \%$ confidence level if the complete CMB dataset is considered. Notice that in the case of the LWB parametrization, the results are closer 


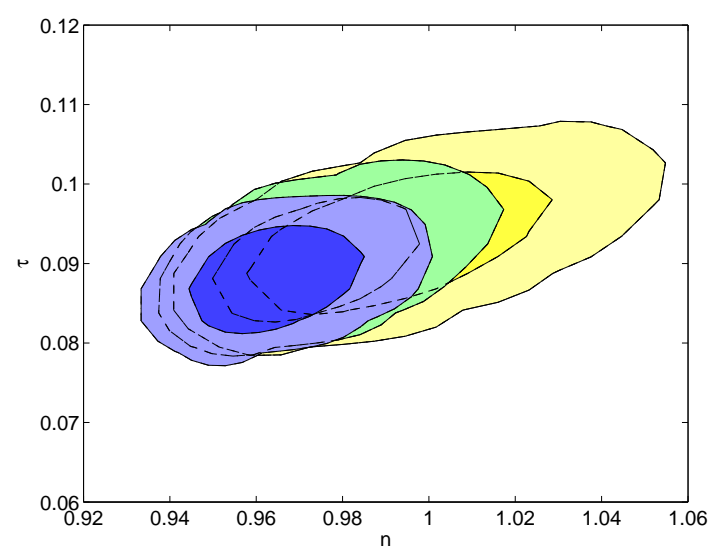

FIG. 1: Contours of the $68 \%$ and $95 \%$ c.l. constraints in the $n$ vs $\tau$ plane for different datasets. The contours regions come from a generalized reionization scenario using (from right to left) the $\mathrm{MH}$ approach from WMAP-7 data (Yellow), "CMB all" (Green), All CMB+BAO (Blue).

to those obtained in the standard sudden reionization scheme. Therefore, the LWB parameterization is less general and samples fewer reionization scenarios than the MH method.

\begin{tabular}{lccc}
\hline \hline Dataset & Ionization & $n(68 \%$ c.l. $)$ & $95 \%$ c.l. \\
\hline \hline WMAP7 & sudden & $0.965 \pm 0.014$ & $n \leq 0.993$ \\
CMB All & sudden & $0.959 \pm 0.013$ & $n \leq 0.984$ \\
WMAP7 & MH & $0.993 \pm 0.023$ & $n \leq 1.042$ \\
CMB All & MH & $0.975 \pm 0.017$ & $n \leq 1.011$ \\
CMB All+LRG-7 & MH & $0.966 \pm 0.014$ & $n \leq 0.994$ \\
CMB All+BAO & MH & $0.965 \pm 0.014$ & $n \leq 0.995$ \\
CMB All+BAO & MH $+w(z)$ & $0.985 \pm 0.018$ & $n \leq 1.025$ \\
WMAP7 & LWB & $0.977 \pm 0.018$ & $n \leq 1.01$ \\
CMB All & LWB & $0.963 \pm 0.015$ & $n \leq 0.998$ \\
\hline \hline
\end{tabular}

TABLE I: Constraints and upper limits on $n$ from several datasets in different recombination scenarios.

Considering the WMAP7 data alone, the HZ spectrum is at $\Delta(-2 \ln (\mathcal{L}))=0.72$ with respect to the best fit model in the case of a $\mathrm{MH}$ recombination scenario $(\Delta(-2 \ln (\mathcal{L}))=2.2$ when all CMB experiments are considered), compared with $\Delta(-2 \ln (\mathcal{L}))=3.3$ when recombination is sudden $(\Delta(-2 \ln (\mathcal{L}))=5.8$ in case of $\mathrm{CMB}$ All).

It is interesting to consider the constraints on the optical depth $\tau$, derived by integrating $x_{e}(z)$ up to $z=32$. Figure 1 shows the $68 \%$ and $95 \%$ c.l. constraints in the $n$ vs. $\tau$ plane arising from different datasets and assuming the $\mathrm{MH}$ reionization parametrization. As we can see the optical depth is always in the range 0.08-0.11, slightly higher than in the standard analysis but consistent with several physical reionization models. Notice also that the HZ spectrum $(n=1)$ is perfectly compatible with WMAP7. Including more datasets shrinks and shifts the contours towards lower values of $n$, but the $\mathrm{HZ}$ case is always reasonably consistent at the $95 \%$ c.l.. Including BAO data rules out the $\mathrm{HZ}$ spectrum at about 2 standard deviations that is considerably less stringent than in the standard case. Moreover, considering a redshift-dependent equation of state weakens the geometrical probes and $\mathrm{HZ}$ is again inside the $68 \%$ confidence level for the $\mathrm{CMB}+\mathrm{BAO}$ case.

It is important to investigate if a more general reionization scenario with the assumption of $n=1$ could provide a viable cosmology, i.e., if the value of cosmological parameters such as the baryon density or the age of the universe are compatible with complementary cosmological information from Big Bang Nucleosythesis and age constraints. It has been shown, for example, in Ref. 13. that the assumption of an $\mathrm{HZ}$ spectrum in the standard analysis with sudden reionization gives a baryon abundance that is at odds with current bounds from BBN. We have therefore performed an analysis using WMAP7 and assuming $n=1$ for the MH reionization scenario, and the results for the different cosmological parameters are summarized in Table 2.

\begin{tabular}{lc}
\hline \hline Parameter & Constraint (68\% c.l.) \\
\hline \hline$\Omega_{b} h^{2}$ & $0.0234 \pm 0.0004$ \\
$\Omega_{c} h^{2}$ & $0.106 \pm 0.005$ \\
$H_{0}\left(\mathrm{~km} \mathrm{~s}^{-1} \mathrm{Mpc}^{-1}\right)$ & $74.2 \pm 2.1$ \\
Age $(\mathrm{Gyr})$ & $13.6 \pm 0.1$ \\
$\Omega_{\Lambda}$ & $0.765 \pm 0.022$ \\
\hline \hline
\end{tabular}

TABLE II: Constraints on cosmological parameters from WMAP7 assuming a HZ primordial spectrum and a reionization scenario characterized by the MH parametrization.

Notice from the results of Table 2 that the constraints on the remaining cosmological parameters are all viable when compared with independent cosmological observables. Current Big Bang Nucleosynthesis constraints from observations of Deuterium abundances provide a constraint of $\Omega_{b} h^{2}=0.0213 \pm 0.002$ at $95 \%$ c.l. [23], which is compatible with the WMAP7 constraint assuming HZ. The constraints shown in Table 2 on $H_{0}$ are also well in agreement with the recent HST determination $h=0.748 \pm 0.036$ at $68 \%$ c.l. 24]. Figure 2 depicts the constraints from WMAP7 in the $\Omega_{b} h^{2}$ vs. $H_{0}$ plane in a generalized recombination scenario, assuming both a varying $n$ and a fixed $n=1$ scenarios. Notice that fixing $n=1$ implies larger values of both $\Omega_{b} h^{2}$ and $H_{0}$.

In conclusion, the details of the reionization processes in the late universe are not very well known. In the ab- 


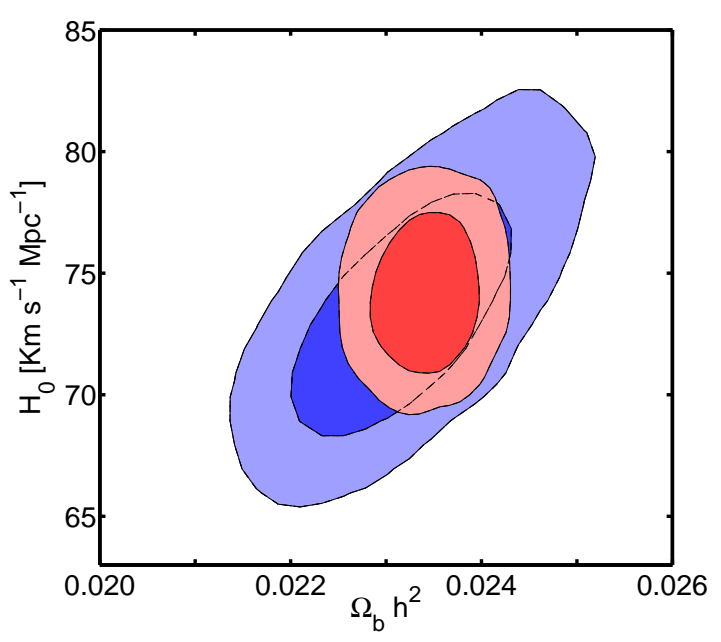

FIG. 2: $68 \%$ and $95 \%$ c.l. WMAP7 constraints in the $H_{0}$ vs. $\Omega_{b} h^{2}$ plane in a generalized reionization scenario described by the $\mathrm{MH}$ parametrization. The larger (blue) contours regions arise when $n$ is allowed to vary. The smaller (red) contours correspond to the HZ $(n=1)$ spectrum.

sence of a precise, full redshift evolution description of the ionization fraction during the reionization period, a simple parametrization, with a single parameter $z_{r}$, has become the standard reionization scheme in numerical analyses. However, more general reionization scenarios are certainly plausible and their impact on the cosmological constraints should be carefully explored. For example, processes of dark matter annihilation as in Ref. [25] could easily be present.

In this paper we have investigated the stability of the CMB constraints on $n$ in generalized reionization scenarios. Our study is motivated by the recent claim that an HZ spectrum is ruled out by current WMAP data at more than $95 \%$ c.l. In agreement with previous studies, we have found that a more general treatment of reionization drastically weakens current CMB constraints on $n$. If only the WMAP 7-year data is considered, a HZ $n=1$ spectrum lies well within the $68 \%$ c.l. allowed region. If all current $\mathrm{CMB}$ datasets are considered in the analysis, the HZ spectrum still lies well within the $95 \%$ c.l. allowed region. If the assumption of constant dark energy is relaxed, $\mathrm{HZ}$ is again inside the $68 \%$ c.l. Therefore, current data do not yet rule out an $\mathrm{HZ}$ spectrum in a conclusive way. It is also worth mentioning that non standard processes during recombination (see e.g., Ref. [26]) or extra relativistic particles (see e.g., Ref. 27]) could be present and could also put $n=1$ in agreement with current observations.

Near future data from the Planck satellite mission are clearly needed to solve this important question. In this respect, performing a parameter analysis with simulated data with the experimental configuration described in Ref. [28], and assuming a model with $n=0.965$, we found that Planck will be able to discriminate it from a $\mathrm{HZ}$ spectrum at more than five standard deviations even in the generalized reionization cases describes here.

[1] J. E. Lidsey, A. R. Liddle, E. W. Kolb, E. J. Copeland, T. Barreiro and M. Abney, Rev. Mod. Phys. 69, 373 (1997).

[2] S. Dodelson, W. H. Kinney and E. W. Kolb, Phys. Rev. D 56, 3207 (1997).

[3] E. Komatsu et al., arXiv:1001.4538 [astro-ph.CO]; D. Larson et al., arXiv:1001.4635 [astro-ph.CO].

[4] W. C. Jones et al., Astrophys. J. 647, 823 (2006); F. Piacentini et al., Astrophys. J. 647, 813 (2006), Astrophys. J. 647, 833 (2006).

[5] M. L. Brown et al. [QUaD collaboration], Astrophys. J. 705, 978 (2009).

[6] C. L. Reichardt et al., Astrophys. J. 694 (2009) 1200.

[7] H. C. Chiang et al., arXiv:0906.1181 [astro-ph.CO].

[8] W. H. Kinney, E. W. Kolb, A. Melchiorri, and A. Riotto, Phys. Rev. D 74, 023502 (2006); W. H. Kinney, E. W. Kolb, A. Melchiorri, and A. Riotto, Phys. Rev. D 78 (2008) 087302.

[9] D. Parkinson, P. Mukherjee, and A. R. Liddle, Phys. Rev. D 73, 123523 (2006).

[10] J. Hamann, S. Hannestad, M. S. Sloth, and Y. Y. Y. Wong, Phys. Rev. D 75, 023522 (2007).

[11] M. Bridges, A. N. Lasenby, and M. P. Hobson, arXiv:astro-ph/0607404

[12] J. Martin and C. Ringeval, JCAP 0608, 009 (2006).

[13] F. Finelli, J. Hamann, S. M. Leach, and J. Lesgourgues, arXiv:0912.0522 [astro-ph.CO].

[14] E. R. Harrison, Phys. Rev. D 1, 2726 (1970); Y. B. Zel'dovich, Mon. Not. Roy. Astron. Soc. 160, (1972), P. J. E. Peebles and J. T. Yu Astrophys. J. 162, 815 (1970).

[15] A. Vallinotto, E. J. Copeland, E. W. Kolb, A. R. Liddle, and D. A. Steer, Phys. Rev. D 69, 103519 (2004).

[16] A. A. Starobinsky, JETP Lett. 82, 169 (2005) [Pisma Zh. Eksp. Teor. Fiz. 82, 187 (2005)].

[17] M. J. Mortonson and W. Hu, Astrophys. J. 686, L53 (2008).

[18] A. Lewis, J. Weller, and R. Battye, Mon. Not. Roy. Astron. Soc. 373, 561 (2006).

[19] A. Lewis, A. Challinor, and A. Lasenby, Astrophys. J. 538 (2000) 473.

[20] A. Lewis and S. Bridle, Phys. Rev. D 66, 103511 (2002).

[21] B. A. Reid et al., arXiv:0907.1659 [astro-ph.CO].

[22] W. J. Percival et al., arXiv:0907.1660 [astro-ph.CO].

[23] M. Pettini, B. J. Zych, M. T. Murphy, A. Lewis, and C. C. Steidel, Mon. Not. Roy. Astron. Soc. 391, 1499 (2008).

[24] A. G. Riess et al., Astrophys. J. Suppl. 183, 109 (2009).

[25] S. Galli, F. Iocco, G. Bertone and A. Melchiorri, Phys. Rev. D 80 (2009) 023505.

[26] S. Galli, R. Bean, A. Melchiorri and J. Silk, Phys. Rev. D 78 (2008) 063532.

[27] R. Bowen, S. H. Hansen, A. Melchiorri, J. Silk and R. Trotta, Mon. Not. Roy. Astron. Soc. 334, 760 (2002); J. Hamann, S. Hannestad, J. Lesgourgues, C. Rampf and Y. Y. Y. Wong, arXiv:1003.3999 [astro-ph.CO].

[28] [Planck Collaboration], arXiv:astro-ph/0604069. 